\title{
Publisher Correction: Ballistic geometric resistance resonances in a single surface of a topological insulator
}

Hubert Maier ${ }^{1}$, Johannes Ziegler ${ }^{1}$, Ralf Fischer ${ }^{1}$, Dmitriy Kozlov²,3, Ze Don Kvon ${ }^{2,3}$, Nikolay Mikhailov², Sergey A. Dvoretsky² \& Dieter Weiss ${ }^{1}$

Correction to: Nature Communications https://doi.org/10.1038/s41467-017-01684-0, published online 08 December 2017

In the original version of this Article, the second and third sentences of the first paragraph of the "Gate voltage and antidot period dependencies" section of the Results originally incorrectly read "The characteristic evolution of the sheet resistance $\rho_{\square}=\rho_{\square}(B=0)$, with $V_{\mathrm{g}}$ shown for three antidot samples and an unpatterned reference sample in Fig. 3a. The maxima of $\rho_{\mathrm{xx}}$, located between $V_{\mathrm{g}} \sim 0.5$ and 1 $\mathrm{V}$, reflect the charge neutrality point (CNP), corresponding to an $E_{\mathrm{F}}$ position located slightly in the valence band (see band structure in Fig. 3b)." In the corrected version, " $\rho_{\square}=\rho_{\square}(B=0)$ " is replaced by " $\rho_{\square}=\rho_{\mathrm{xx}}(B=0)$ ", and "The maxima of $\rho_{\mathrm{xx}}$ " is replaced by "The maxima of $\rho_{\square} "$.

These errors have been corrected in both the PDF and HTML versions of the Article.

Published online: 05 February 2018

(i) Open Access This article is licensed under a Creative Commons Attribution 4.0 International License, which permits use, sharing, adaptation, distribution and reproduction in any medium or format, as long as you give appropriate credit to the original author(s) and the source, provide a link to the Creative Commons license,

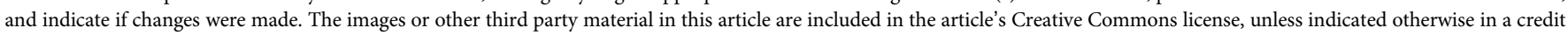
line to the material. If material is not included in the article's Creative Commons license and your intended use is not permitted by statutory regulation or exceeds the permitted use, you will need to obtain permission directly from the copyright holder. To view a copy of this license, visit http://creativecommons.org/licenses/by/4.0/.

(C) The Author(s) 2018

\footnotetext{
${ }^{1}$ Institute of Experimental and Applied Physics, University of Regensburg, 93053 Regensburg, Germany. ${ }^{2}$ A.V. Rzhanov Institute of Semiconductor Physics, 630090 Novosibirsk, Russia. ${ }^{3}$ Novosibirsk State University, 630090 Novosibirsk, Russia Correspondence and requests for materials should be addressed to D.W. (email: dieter.weiss@ur.de)
} 